\section{Andrógenos e SHBG: Em Busca do Elo Desconbecido}

A UNIDADE PILO-SEbÁCEA é um órgão alvo para os andrógenos e a exposição desta estrutura a uma concentração aumentada de andrógenos é a base da fisiopatologia das muitas manifestações das síndromes hiperandrogênicas, independentemente da etiologia. Entretanto, sinais e sintomas de hiperandrogenismo, como pubarca, hirsutismo, alopecia e anovulação, podem estar presentes em uma paciente com concentração normal de andrógenos, enquanto um aumento da sua produção não se acompanha necessariamente das características clínicas de excesso androgênico (1). Estes dados demonstram que eventos teciduais são fatores importantes na determinação da sensibilidade cutânea aos andrógenos (2).

Tem sido demonstrado que meninas com pubarca precoce decorrente de adrenarca precoce e mulheres com hirsutismo podem apresentar concentração sérica elevada de $3 \alpha$-androstanediol glicuronídeo ( $3 \alpha$-diol G), um metabólito da dihidrotestosterona (DHT), e do androstanediol, originados da conversão destes por ação da $5 \alpha$-redutase. Mulheres hirsutas têm um aumento da atividade da $5 \alpha$-redutase na pele, quando comparadas a mulheres não hirsutas, e esta enzima parece ser um dos principais moduladores da sensibilidade cutânea aos andrógenos. Entretanto, a produção da DHT é um processo localizado, e sua concentração sérica não se correlaciona necessariamente com a presença e intensidade das manifestações hiperandrogênicas. Por outro lado, a DHT é convertida a androstanediol, que é glicuronidado por ação de uma glicuronil-transferase e liberado na circulação, dando origem ao $3 \alpha$-diol G.

Este tem sido considerado um marcador da ação biológica dos andrógenos sobre a unidade pilo-sebácea. Entretanto, existem evidências que a atividade glicuronil-transferase é muito maior no fígado do que na pele, o que pode significar que, apesar da DHT e do androstanediol serem convertidos a $3 \alpha$-diol $G$ no tecido periférico, o principal local de glicuronidação dos androgênios seja no fígado. Além do mais, alguns autores consideram que a concentração sérica do $3 \alpha$-diol G reflita mais uma produção de andrógenos adrenais. Rittmaster e cols (3) demonstraram que os precursores do $3 \alpha$-diol $\mathrm{G}$ em mulheres hirsutas originam-se quase que exclusivamente da adrenal, enquanto Vermeulen e Giagulli (4), a partir de dados obtidos da infusão contínua de esteróides em mulheres normais, demonstraram que o $3 \alpha$-diol $\mathrm{G}$ se origina quase que exclusivamente a partir da androstenediona, DHEA e DHEAS. Entretanto, Duffy e cols (5) demonstraram, através da administração de DHT por duas vias diferentes (endovenosa e subcutânea), que a pele é o principal local da produção de $3 \alpha$-diol e de $3 \alpha$-diol G, com este último refletindo principalmente o metabolismo da DHT.

Outros fatores devem ser levados em consideração na tentativa de compreender a fisiopatologia das manifestações clínicas das síndromes hiperandrogênicas. Dentre vários, o papel da globulina ligadora dos esteróides sexuais (SHBG) merece um lugar de destaque.

\section{editorial}

\author{
José Antonio M. Marcondes
}


Aproximadamente $96 \%$ da testosterona plasmática e de outros $17 \beta$-hidroxiesteróides circulam ligados a proteínas carregadoras. A SHBG e a albumina são as principais proteínas ligantes de andrógenos no plasma. A SHBG é uma glicoproteína sintetizada no fígado, cuja síntese é modulada por vários fatores fisiológicos e patológicos. Estrógenos e hormônios tireoidianos estimulam sua síntese, enquanto andrógenos, glicocorticóides, hormônio de crescimento e insulina a inibem (6). Uma vez que a SHBG é freqüentemente diminuída nas síndromes hiperandrogênias, a concentração de testosterona livre apresenta-se elevada em mulheres cuja testosterona total é normal.

Enquanto na mulher adulta não existe consenso sobre a importância e a interrelação entre os andrógenos e seus metabólitos periféricos, na idade pré-puberal praticamente inexistem dados que permitam a formulação de uma hipótese aceitável. A esse respeito, é interessante o modelo de estudo adotado por Teixeira e cols neste número dos Arquivos (7), para avaliar o papel dos androgênios adrenais e periféricos na modulação da concentração da SHBG na infância. Meninas com pubarca precoce tendo por etiologia a adrenarca precoce foram comparadas com meninas prépúberes, pareadas para a mesma idade. $\mathrm{O}$ grupo de meninas com pubarca precoce apresentou concentração de SHBG significativamente menor e de DHEAS significativamente maior que as do grupo controle, tendo-se observado que a SHBG se correlacionou inversamente com o IMC e com o $3 \alpha$-diol G. Estes mesmos autores observaram, em estudo em que avaliaram apenas meninas com pubarca precoce (8), uma correlação inversa entre a concentração de SHBG com IGF-l e IGFBP-1. A interpretação dada a esses achados pelos autores é que o eixo insulina/ IGFI/IGFBP-1 seria um importante regulador da concentração da SHBG em meninas com pubarca precoce.

De fato, os dados do estudo publicado neste número dos Arquivos também apontam, indiretamente, para uma influência da insulina sobre a concentração da SHBG. A correlação inversa observada entre o IMC e a SHBG é compatível com uma diminuição da sensibilidade à insulina (induzida pelos andrógenos?) possivelmente por sua ação sobre a composição corporal. A esse respeito, cumpre lembrar os trabalhos de Ibañez e cols $(9,10)$, sobre recém-nascido de baixo peso, diminuição da sensibilidade à insulina, pubarca precoce e síndrome dos ovários policísticos.

Seria mais uma vez a resistência à insulina culpada? Embora este não tenha sido o objetivo do trabalho de Teixeira e cols, os dados existentes na literatura parecem apontar que sim.

\section{REFERÊNCIAS}

1. Kirschner MA, Jacobs JB. Combined ovarian and adrenal vein catheterization to determine the site(s) of androgen overproduction in hirsute women. J Clin Endocrinol Metab 1971;33:199-209.

2. Lobo R, Goebelsman U, Horton R. Evidence for the importance of peripheral tissues events in the development of hirsutism in polycystic ovary syndrome. J Clin Endocrinol Metab 1983;57:393-7.

3. Rittmaster RS, Zwicker H, Thompson DL, Konok G, Norman RW. Androstanediol glucuronide production in human liver, prostate, and skin. Evidence for the importance of the liver in 5a-reductase androgen metabolism. J Clin Endocrinol Metab 1993;76:997-82.

4. Vermeulen A, Giagulli VA. Physiopathology of plasma androstanediol-glucuronide. J Steroid Biochem Molec Biol 1991;39:829-33.

5. Duffy DM, Legro RS, Chang L, Stancsy K, Lobo RA. Metabolism of dihydrotestosterone to 5a-androstane$3 a, 17 b-d i o l$ glucuronide is greater in the peripheral compartment than in the splanchnic compartment. Fertil Steril 1995;64:736-9.

6. Nestler J, Powers L, Mah DW. A direct effect of hyperinsulinemia on serum sex hormone-binding globulin levels in obese women with PCO syndrome. J Clin Endocrinol Metab 1991;72:83-9.

7. Teixeira RJ, Dimetz T, Bordallo MA, Guimarães MM. Papel dos androgênios adrenais e periféricos na modulação dos níveis da globulina ligadora dos hormônios sexuais na pubarca precoce. Arq Bras Endocrinol Metab 2002;46/5: - .

8. Teixeira RJ, Silva VCG, Gazolla HM, Cunha SB, Guimarães MM. The relationship between ovarian structure and serum insulin, insulin-like growth factor-I (IGF-I) and its binding protein (IGFBP-1 and IGFBP-3) levels in premature pubarche. J Pediatr Endocrinol Metab 2002; 15:69-75.

9. Ibanez L, Valls C, Potau N, Marcos MV, de Zegher F. Polycystic ovary syndrome after precocious pubarche: ontogeny of the low-birth weight effect. Clin Endocrinol (Oxf) 2001:55:667-72.

10. Ibanez L, Potau N, de Zegher F. Recognition of a new association: reduced fetal growth, precocious pubarche, hyperinsulinism and ovarian dysfunction. Ann Endocrinol (Paris) 2000;61:141-2. 